\title{
EICHLER-SHIMURA HOMOLOGY, INTERSECTION NUMBERS AND RATIONAL STRUCTURES ON SPACES OF MODULAR FORMS
}

\author{
SVETLANA KATOK AND JOHN J. MILLSON \\ Dedicated to our friend Boris Weisfeiler
}

\begin{abstract}
In this paper we reinterpret the main results of $[8]$ using the intersection theory of cycles with coefficients. To this end we give a functorial interpretation of Eichler-Schimura periods.
\end{abstract}

Introduction. In this paper we reformulate the main results of S. Katok [8] in terms of homology theory with local coefficients. In order to do so we first give an interpretation of the period of a cusp form $f$ of weight $2 m+2(m \geq 1)$ over a closed geodesic $[\gamma]$ as the Kronecker index of the cohomology class Sh $f$ with local coefficients associated to $f$ by Shimura $[\mathbf{1 3}]$ and a 1-cycle with dual local coefficients associated to $[\gamma]$. We will call this period the Eichler-Shimura period. There are other interpretations of Eichler-Shimura periods but these seem to work only for arithmetic fundamental groups whereas the defining integral of the period makes sense quite generally.

There is a simple idea from group homology theory which is the basis of our reformulation - that of a "decomposable" cycle. We now give the definition.

Suppose $\sigma$ is a representation of a group $\Gamma$ into $G L(V)$ where $V$ is a finite dimensional vector space. Then we may consider the Eilenberg-Mac Lane homology group $H_{1}(\Gamma, \sigma)$. We observe that if $\gamma$ is an element of $\Gamma$ and $v$ is an element of $V$ satisfying $\sigma(\gamma) v=v$ (a $\gamma$-invariant) then the 1-chain $\gamma \otimes v$ satisfies

$$
\partial(\gamma \otimes v)=\sigma(\gamma) v-v=0 .
$$

Consequently, $\gamma \otimes v$ is a 1-cycle and gives rise to an element of $H_{1}(\Gamma, \sigma)$ which we also denote $\gamma \otimes v$.

DEFINITIONS. We will call such cycles decomposable cycles and we will say a homology class is decomposable if it has a decomposable representative.

REMARK. A general 1-cycle is of the form $\sum_{j=1}^{n} \gamma_{j} \otimes v_{j}$ where

$$
\sum_{j=1}^{n} \sigma\left(\gamma_{j}\right) v_{j}-\sum_{j=1}^{n} v_{j}=0
$$

For more details on homology and cohomology of groups see Brown $[\mathbf{3}]$.

We can now give our formulation of Eichler-Shimura periods. Let $V$ be the space of the standard 2-dimensional complex representation of $S L_{2}(\mathbf{R})$ and $V^{*}$ be its dual. The corresponding real space we will denote by $V_{\mathbf{R}}$. We let $S^{m} V$ denote

Received by the editors January 31, 1986.

1980 Mathematics Subject Classification (1985 Revision). Primary 11F06, 20H10, 55N25; Secondary $22 \mathrm{E} 40$.

The second author was partially supported by an NSF grant.

(C) 1987 American Mathematical Society $0002-9947 / 87 \$ 1.00+\$ .25$ per page 
the $m$ th symmetric power of $V$. We let $f$ be an element of $S_{2 m+2}(\Gamma)$, the space of holomorphic cusp forms of weight $2 m+2$ for a Fuchsian group $\Gamma$ of finite covolume in $S L_{2}(\mathbf{R})$. In most of what follows $m$ will play no role and we will abbreviate $S^{2 m} V$ by $E$ and the underlying real space by $E_{\mathbf{R}}$. Then we have the embedding of Shimura $[\mathbf{1 3}]$ :

$$
\text { Sh: } S_{2 m+2}(\Gamma) \rightarrow H^{1}(\Gamma, E) \text {. }
$$

Let $\langle\langle\rangle$,$\rangle denote the Kronecker pairing of H^{1}(\Gamma, E)$ with $H_{1}\left(\Gamma, E^{*}\right)$ and let us denote the standard basis for the space $V^{*}$ by $\{u, v\}$. As a model for $E^{*}$ we consider the space of all homogeneous polynomials of degree $2 m$ in $u$ and $v$ with complex coefficients. The group $G L_{2}(\mathbf{R})$ acts on $E^{*}$ by the following formula

$$
\rho(g) P_{2 m}(u, v)=P_{2 m}(a u+b v, c u+d v),
$$

where $g^{-1}=\left[\begin{array}{ll}a & b \\ c & d\end{array}\right]$ and $P_{2 m}(u, v) \in E^{*}$. Suppose now $\gamma=\left[\begin{array}{ll}a & b \\ c & d\end{array}\right]$ is a hyperbolic element of $\Gamma$. Then the 1-chain

$$
\gamma \otimes\left(c u^{2}+(d-a) u v-b v^{2}\right)^{m}
$$

is a decomposable cycle and we have the following result, Theorem 2.1 of this paper.

THEOREM.

$$
\left\langle\left\langle\operatorname{Sh} f, \gamma \otimes\left(c u^{2}+(d-a) u v-b v^{2}\right)^{m}\right\rangle\right\rangle=\int_{z_{0}}^{\gamma z_{0}} f(z)\left(c z^{2}+(d-a) z-b\right)^{m} d z .
$$

Here $z_{0}$ is any point in the upper half-plane $\mathbf{H}$ and the right-hand side is the integral along any path joining $z_{0}$ and $\gamma z_{0}$ in $\mathbf{H}$-this is the Eichler-Shimura period. The proof of this formula depends on a de Rham interpretation of $H^{1}(\Gamma, E)$ and a simplicial interpretation of $H_{1}\left(\Gamma, E^{*}\right)$.

Now if $\Theta_{m+1, \gamma}$ denotes the relative (or hyperbolic) Poincaré series given in (1.3) of [8] we have that the Petersson inner product $\left(\left(f, \Theta_{m+1, \gamma}\right)\right)_{2 m+2}$ is the above Eichler-Shimura period. We find as a consequence of the previous formula the following theorem-see Theorem 4.2 of the text.

THEOREM. The relative (hyperbolic) Poincaré series $\Theta_{m+1, \gamma}$ span $S_{2 m+2}(\Gamma)$ as a real vector space if and only if the decomposable classes span $H_{1}\left(\Gamma, E_{\mathbf{R}}^{*}\right)$.

As a consequence the result of $\mathrm{S}$. Katok from [8] may be restated as saying that the decomposable classes span $H_{1}\left(\Gamma, E_{\mathbf{R}}^{*}\right)$. We observe that this homological reformulation does not require the existence of a quotient $\Gamma \backslash \mathbf{H}$. It makes sense for arbitrary subgroups $\Gamma$ of $S L_{2}(\mathbf{R})$. Our reformulation is to some degree justified by the fact that Goldman and Millson [5] have proved this algebraic version for any finitely generated, Zariski-dense subgroup of $S L_{2}(\mathbf{R})$. This reformulation also plays a critical role in their construction of an explicit finite spanning set of hyperbolic Poincaré series for any Fuchsian group of finite covolume. However, we did not have this result as our primary goal in making this reformulation but rather we had higher dimensional generalizations in mind. Here one must replace the cycles $\gamma \otimes v$ with the cycles considered in Johnson and Millson [7].

In $\S \S 5$ and 6 we restrict to certain arithmetic groups $\Gamma$ and verify that the rational structures on $S_{2 m+2}(\Gamma)$ constructed by S. Katok [8] coincide with the usual ones coming from Eichler-Shimura theory. The rational structures coming from EichlerShimura theory are described in detail in $\S 5$ and compared to those of S. Katok in $\S 6$. 
In the final section of our paper we give a reformulation of the period formula, Theorem 3, of S. Katok [8] in terms of an intersection product of decomposable cycles $\gamma_{1} \otimes v_{1} \cdot \gamma_{2} \otimes v_{2}$. Such an intersection product is a sum over the points of $\gamma_{1} \cap \gamma_{2}$ of an intersection multiplicity multiplied by a coefficient $\left(v_{1}, v_{2}\right)$. This coefficient contribution is the inner product of two elements of $E^{*}$ using the natural symmetric form.

We thank Bill Goldman for some interesting discussions concerning the Shimura isomorphism. Our discussion of the Shimura isomorphism was influenced by Chapter 2 of his paper [4]. We also thank John Stembridge for useful conversations.

1. The Shimura isomorphism. In this section we review the construction of the Shimura isomorphism $[\mathbf{1 3}]$ in the case of even weight forms. Let $N$ be a manifold and $G$ a Lie group which operates on $N$ from the left. Let $\chi(N)$ denote the smooth vector fields on $N$ and $\mathfrak{g}$ denote the Lie algebra of $G$. Then there is a linear map $\Phi$ from $\mathfrak{g}$ to $\chi(N)$ defined as follows. Let $v \in \mathfrak{g}$. Then $\Phi(v)=V$ where if $x \in N$ and $f$ is a smooth function defined near $x$ we have:

$$
V f(x)=\left.\frac{d}{d t} f(\exp (-t v) x)\right|_{t=0}
$$

We extend $\Phi$ to a map from the complexification $\mathfrak{g}_{\mathbf{C}}$ of $\mathfrak{g}$ to complex vector fields on $N$. If $N$ is a complex manifold with almost complex structure $J$ and $G$ preserves $J$ then we may replace $\Phi$ by $\Phi^{\prime}$ with

$$
\Phi^{\prime}=-(\Phi-i J \circ \Phi) \text {. }
$$

Then $\Phi^{\prime}$ takes values in the holomorphic vector fields on $N$. We observe that $\Phi$ and $\Phi^{\prime}$ are $G$-homomorphisms.

The hypotheses of the above paragraph are satisfied in case $N$ is the upper halfplane, to be denoted $\mathbf{H}$, and $G=P S L_{2}(\mathbf{R})$. For future reference we observe that if we identify $\mathbf{H}$ with $P S L_{2}(\mathbf{R}) / S O_{2}$ then the usual complex structure on $\mathbf{H}$ coincides with the $G$-invariant complex structure whose value at the identity coset is induced by the quotient of the action of $J_{0}$ on $\mathfrak{g}$ by $x \rightarrow\left[J_{0}, x\right]$ where $J_{0}=\frac{1}{2}\left[\begin{array}{rr}0 & 1 \\ -1 & 0\end{array}\right]$. We find the formula

$$
\Phi^{\prime}\left(\left[\begin{array}{rr}
a & b \\
c & -a
\end{array}\right]\right)=\left(-c z^{2}+2 a z+b\right) \frac{\partial}{\partial z} .
$$

In particular $\Phi^{\prime}$ takes values in $\mathcal{Q}$, the space of quadratic vector fields on $\mathbf{H}$.

It is convenient to observe that $\mathfrak{g}_{\mathbf{C}}$ is equivalent as a $G$-module to $S^{2} V^{*}$. This equivalence is obtained by using the symplectic form $($,$) on V$ given by $(e, f)=1$ where $\{e, f\}$ is the standard basis for the space $V$. If $A \in \mathfrak{g}$, we define $s_{A} \in S^{2} V^{*}$ by $s_{A}\left(v_{1}, v_{2}\right)=\left(A v_{1}, v_{2}\right)$. Letting $\{u, v\}$ be a basis for $V^{*}$ dual to $\{e, f\}$ we obtain the following formula for $\Phi^{\prime}$ transported to $S^{2} V^{*}$ which we denote $\Psi$ :

$$
\Psi\left(a u^{2}+b u v+c v^{2}\right)=\left(a z^{2}+b z+c\right) \frac{\partial}{\partial z} .
$$

For every natural number $m, \Psi$ induces a $G$-homomorphism

$$
S^{m} \Psi: S^{m} S^{2} V^{*} \rightarrow S^{m} \mathcal{Q} .
$$

We may also regard $S^{m} \Psi$ as a $G$-invariant element of $S^{m} S^{2} V \otimes S^{m} \mathcal{Q}$ and also as a $G$-invariant global section of the sheaf $\overparen{S^{m} S^{2} V} \otimes S^{m} \Theta$ where $\Theta$ is the sheaf of 
germs of holomorphic vector fields on $\mathbf{H}$ and $\overparen{S^{m} S^{2} V}$ is the sheaf of locally constant functions on $\mathbf{H}$ with values in $S^{m} S^{2} V$.

We now consider the projection $p: S^{m} S^{2} V^{*} \rightarrow E^{*}$. One way of viewing $p$ is as follows. We embed $S^{m} S^{2} V^{*}$ in $\bigotimes_{1}^{m} S^{2} V^{*}$. This latter space can be realized as products of quadratic forms in $m$ distinct sets of variables $\left(u_{1}, v_{1}\right),\left(u_{2}, v_{2}\right), \ldots$, $\left(u_{m}, v_{m}\right)$. Precisely we can realize $\bigotimes_{1}^{m} S^{2} V^{*}$ as functions on $\bigoplus_{1}^{m} V$ as indicated. The projection $p$ may then be realized by restriction to the diagonal. We will need another realization of $p$ in $\S 5$. The space $S^{m} S^{2} V^{*}$ is the space of all homogeneous polynomials of degree $m$ in three variables each of which is a quadratic polynomial in two variables. The projection $p$ may also be realized by substitution: it projects each homogeneous polynomial of degree $m$ in three variables onto a homogeneous polynomial of degree $2 m$ in two variables.

We have a similar projection $q: S^{m} \mathcal{Q} \rightarrow \mathcal{P}$ where $\mathcal{P}$ is the space of $m$-vector fields on $\mathbf{H}$ of the form $p(z) \partial^{\otimes m} / \partial z$ where $p(z)$ is a polynomial of degree less than or equal to $2 m$. The projection $q$ may be constructed as follows. We embed $S^{m} \mathcal{Q}$ in the space of sections of the $m$-fold external tensor product $T^{\prime}\left(\mathbf{P}^{1}\right) \otimes \cdots \otimes T^{\prime}\left(\mathbf{P}^{1}\right)$. Here the prime denotes the holomorphic tangent bundle. We recall that the external tensor product $E \otimes F$ of two bundles $E$ and $F$ over $X$ and $Y$ respectively assigns to $(x, y)$ the tensor product of the fibers $E_{X}$ and $E_{Y}$. The diagonal map $\Delta: \mathbf{P}^{1} \rightarrow\left(\mathbf{P}^{1}\right)^{m}$ induces a bundle map:

$$
\Delta^{*}: T^{\prime}\left(\mathbf{P}^{1}\right) \otimes \cdots \otimes T^{\prime}\left(\mathbf{P}^{1}\right) \rightarrow T^{\prime \otimes m}\left(\mathbf{P}^{1}\right) .
$$

Then $\Delta^{*}$ restricted to $S^{m} \mathcal{Q}$ gives rise to the projection $q$. We observe that the image of $S^{m} \mathcal{Q}$ under $\Delta^{*}$ is $\mathcal{P}$. It is clear that $q \circ S^{m} \Psi$ factors through $p$ and gives rise to a $G$-homomorphism $S$ from $E^{*}$ to $P$.

LEMMA 1.1 .

$$
S\left(\sum_{j=0}^{2 m} a_{j} u^{j} v^{2 m-j}\right)=\left(\sum_{j=0}^{2 m} a_{j} z^{j}\right) \frac{\partial^{\otimes m}}{\partial z} .
$$

PROOF. It is sufficient to prove that $S\left(u^{j} v^{2 m-j}\right)=z^{j} \partial^{\otimes m} / \partial z$. We lift $u^{j} v^{2 m-j}$ back to $\tau=u_{1} v_{1} \otimes \cdots \otimes u_{j} v_{j} \otimes v_{j+1}^{2} \otimes \cdots \otimes v_{m}^{2}$ in $\otimes_{1}^{m} S^{2} V^{*}$. The image of $\tau$ under $S^{m} \Psi$ is $\nu=z_{1} \partial / \partial z_{1} \otimes \cdots \otimes z_{j} \partial / \partial z_{j} \otimes \partial / \partial z_{j+1} \otimes \cdots \otimes \partial / \partial z_{m}$. But $\Delta^{*}(\nu)=z^{j} \partial^{\otimes m} / \partial z$ and the lemma is proved.

LEMMA 1.2. Under the isomorphism between $\operatorname{Hom}\left(E^{*}, \mathcal{P}\right)$ and $E \otimes P$ the image of $S$ (again denoted $S$ ) is given by

$$
S=\sum_{j=0}^{2 m}\left(\begin{array}{c}
2 m \\
j
\end{array}\right) e^{j} f^{2 m-j} \otimes z^{j} \frac{\partial^{\otimes m}}{\partial z} .
$$

ProOF. We have only to check that the basis $\left\{\left(\begin{array}{c}2 m \\ j\end{array}\right) e^{j} f^{2 m-j}, 1 \leq j \leq 2 m\right\}$ is dual to the basis $\left\{u^{j} v^{2 m-j}\right\}$ and the lemma is proved.

We now regard $S$ as a $G$-invariant section of the sheaf $\tilde{E} \otimes \Theta^{m}$. If $\mathcal{K}$ is the sheaf of germs of holomorphic 1-forms on $\mathbf{H}$ then contraction with $S$ gives a $G$-map of sheaves, to be denoted Sh:

$$
\mathrm{Sh}: K^{m+1} \rightarrow K \otimes \tilde{E} .
$$

We have the following lemma. 
LEMMA 1.3. If $f(z) d z^{m+1}$ is the germ of a holomorphic section of $\mathcal{K}^{m+1}$ at a point $z_{0}$ in $\mathbf{H}$, then

$$
\operatorname{Sh}\left(f(z) d z^{m+1}\right)=\sum_{j=0}^{2 m}\left(\begin{array}{c}
2 m \\
j
\end{array}\right) \tilde{e}^{j} \tilde{f}^{2 m-j} \otimes z^{j} f(z) d z .
$$

Notation. If $s$ is an element of $E$ we will let $\tilde{s}$ denote the constant function on $\mathbf{H}$ taking $s$ as a value.

We may rewrite the right-hand side of the above formula as

$$
\operatorname{Sh}\left(f(z) d z^{m+1}\right)=(z \tilde{e}+\tilde{f})^{2 m} \otimes f(z) d z .
$$

This new expression has a rather different meaning from the previous one since $(z \tilde{e}+\tilde{f})^{2 m}$ is not a constant function on $\mathbf{H}$ with values in $E$. It is a section of a homogeneous line bundle $\mathcal{L}$ - over $\mathbf{H}$. Though this point of view will play no role in what follows we make a digression to give some details since it is in this form that $\operatorname{Sh}\left(f(z) d z^{m+1}\right)$ is usually given.

The flat bundle $\tilde{E}$ is a direct sum of homogeneous line bundles. Indeed the action of the maximal compact subgroup $S_{2}$ of $S L_{2}(\mathbf{R})$ on $E$ may be diagonalized and this diagonalization breaks $\tilde{E}$ into a sum of homogeneous line bundles. Recall $J_{0}=\frac{1}{2}\left[\begin{array}{rr}0 & 1 \\ -1 & 0\end{array}\right]$. The action of $J_{0}$ on $\mathfrak{P}$ (the orthogonal complement of the Lie algebra of $S_{2}$ in $\mathfrak{g}$ ) as a derivation induces the almost complex structure on the tangent space to $\mathbf{H}$ at the identity coset of $S L_{2}(\mathbf{R}) / S O_{2}$. Also the above decomposition of $E$ is the decomposition of $E$ into eigenspaces under the derivation action of $J_{0}$. The eigenvalues are pure imaginary and we order them in the obvious way. We let $L_{-}$be the lowest (that is the $-2 m i$ ) eigenspace for the action of $J_{0}$ and $\mathcal{L}_{-}$be the corresponding homogeneous line bundle. The connection with the above formula is the following.

LEMMA 1.4. The fiber of $\mathcal{L}_{-}$over $z$ is generated by $(z e+f)^{2 m}$.

PROOF. We observe that we may realize $\mathbf{H}$ as the space of negative definite complex structures on $V_{\mathbf{R}}$ (we say $J$ is negative definite if the form $s_{J}\left(v_{1}, v_{2}\right)=$ $\left(v_{1}, J v_{2}\right)$ is negative definite for $\left.v_{1}, v_{2} \in V_{\mathbf{R}}\right)$. Under this correspondence $J=\left[\begin{array}{ll}a & b \\ c & d\end{array}\right]$ corresponds to the point $z=-d / c-i / c$ (note $c<0$ ). But then the $-i$ eigenspace of $J$ on $V$ is generated by $z e+f$ and the $-2 m i$ eigenspace of $J$ on $E$ is generated by $(z e+f)^{2 m}$.

We now return to our goal of expressing Eichler-Shimura integrals as a Kronecker index.

Let $\xi=\sum_{j=0}^{2 m} a_{j} u^{j} v^{2 m-j}$ be an element of $E^{*}$ and let $\tilde{\xi}$ be the corresponding constant section of $\tilde{E}^{*}$. Then we have the following lemma.

LEMMA 1.5 .

$$
\left\langle\operatorname{Sh}\left(f(z) d z^{m+1}\right), \tilde{\xi}(z)\right\rangle=f(z)\left(\sum_{j=0}^{2 m} a_{j} z^{j}\right) d z .
$$

In particular if $\xi=\left(a u^{2}+b u v+c v^{2}\right)^{m}$ we have

$$
\left\langle\operatorname{Sh}\left(f(z) d z^{m+1}\right), \tilde{\xi}(z)\right\rangle=f(z)\left(a z^{2}+b z+c\right)^{m} d z .
$$


Notation. Henceforth we will use $\langle$,$\rangle as a generic symbol for the pairing between$ a space and its dual. In the case above $\langle$,$\rangle denotes the pairing between E$ and $E^{*}$ (considered as fibers over $z$ of the associated product bundles).

Now suppose $\Gamma$ is a torsion free discrete group acting properly discontinuously on $\mathbf{H}$ with quotient $M=\Gamma \backslash \mathbf{H}$ (we do not assume $M$ has finite volume). Since $\mathrm{Sh}$ is a $\Gamma$-map it induces maps of the sheaf cohomology groups on $M$ associated to $K^{m+1}$ and $\mathcal{K} \otimes \tilde{E}$ where $\mathcal{K}$ and $\tilde{E}$ now denote the induced bundles on $M$. In particular we get a map

$$
\mathrm{Sh}: H^{0}\left(M, \mathcal{K}^{m+1}\right) \rightarrow H^{0}(M, \mathcal{K} \otimes \tilde{E}) .
$$

An element of $H^{0}(M, \mathcal{K} \otimes \tilde{E})$ is a fortiori a closed 1-form with values in $\tilde{E}$. We recall that a 1-form $\omega$ with values in a bundle $E$ over $M$ assigns to every tangent vector $v \in T_{x}(M)$ an element $\omega(v)$ in $E_{x}$, the fiber of $E$ over $x$. Let us recall the map from closed 1 -forms with values in $\tilde{E}$ to simplicial cochains on $M$ with coefficients in $\tilde{E}$. Let $\left(v_{1}, v_{2}\right) \otimes s$ be a simplicial 1-chain with coefficients in $\tilde{E}^{*}$ so $\left(v_{1}, v_{2}\right)$ is an oriented 1 -simplex of $M$ and $s$ is a parallel section of $\tilde{E}^{*}$ restricted to $\left(v_{1}, v_{2}\right)$. Then the $\left\langle\omega \mid\left(v_{1}, v_{2}\right), s\right\rangle$ is a scalar 1-form on $\left(v_{1}, v_{2}\right)$. We define

$$
\left\langle\left\langle\omega,\left(v_{1}, v_{2}\right) \otimes s\right\rangle\right\rangle=\int_{\left(v_{1}, v_{2}\right)}\langle\omega, s\rangle .
$$

In this way we obtain a map from the de Rham cohomology of $\tilde{E}$ valued forms to the simplicial cohomology with coefficients in $\tilde{E}$. By the de Rham theorem this map is an isomorphism. We refer to Johnson and Millson [7, §4], for details.

From the preceding discussion we obtain a map $H^{0}(M, \mathcal{K} \otimes \tilde{E}) \rightarrow H^{1}(M, \tilde{E})$. Composing with Sh we obtain a map again denoted by Sh:

$$
\mathrm{Sh}: H^{0}\left(M, \mathcal{K}^{m+1}\right) \rightarrow H^{1}(M, \tilde{E}) .
$$

From the local formula in Lemma 1.3 we obtain the following formula for the map Sh.

LEMMA 1.6. Let $f(z) d z^{m+1}$ be an element of $H^{0}\left(M, K^{m+1}\right)$ (identified with a $\Gamma$-invariant on $\mathbf{H})$. Then $\mathrm{Sh} f$ is represented in de Rham cohomology by the 1-form $\omega_{f}$ (with values in $\left.\tilde{E}\right)$ given by

$$
\omega_{f}=\sum_{j=0}^{2 m}\left(\begin{array}{c}
2 m \\
j
\end{array}\right) \tilde{e}^{j} \tilde{f}^{2 m-j} \otimes z^{j} f(z) d z .
$$

REMARKS. 1. In case $M$ is compact so there is a good theory of harmonic forms (see $\S 3$ ), then $\omega_{f}$ is harmonic. Indeed we have seen that we may rewrite

$$
\omega_{f}=(z \tilde{e}+\tilde{f})^{2 m} \otimes f(z) d z .
$$

From this expression and Murakami [11, Lemma 5.4], we see that $\omega_{f}$ is harmonic. Conversely, the above lemma of Murakami implies that any harmonic $(1,0)$ form with values in $\tilde{E}$ must take values in the subbundle $\mathcal{L}_{-}$and consequently is essentially of the above form.

2. In the finite volume case if $f$ is cuspidal then $\omega_{f}$ is a square integrable harmonic form. 
We now discuss the relationship between Sh, group cohomology and "period polynomials". Since $M$ is a manifold of type $K(\Gamma, 1)$ we may regard $H^{1}(M, \tilde{E})$ as a model for the group cohomology $H^{1}(\Gamma, E)$. Also $H^{0}\left(M, K^{m+1}\right)$ is isomorphic to $M_{2 m+2}(\Gamma)$, the space of (holomorphic) modular forms of weight $2 m+2$. Thus we may write $\mathrm{Sh}$ as a map

$$
\text { Sh: } M_{2 m+2}(\Gamma) \rightarrow H^{1}(\Gamma, E) .
$$

In order to obtain the period polynomials we recall that a representing EilenbergMac Lane cocycle for an element $\alpha$ in $H^{1}(\Gamma, E)$ is a crossed homomorphism from $\Gamma$ to $E$. Thus to obtain the crossed homomorphism associated to $f \in S_{2 m+2}$ by the Shimura isomorphism it is necessary to give an explicit isomorphism from $H^{1}(M, \tilde{E})$ to $H^{1}(\Gamma, E)$. We think about the first group as simplicial cohomology. We recall the map-see Johnson and Millson [7, §4]. Let $\alpha \in H^{1}(M, \tilde{E})$, let $m_{0}$ be the base point for $M$ and let $c=\sum_{j=1}^{n} s_{j}$ be an edge-path of oriented 1-simplices beginning and ending at $m_{0}$. Let $a$ be a simplicial cocycle representing $\alpha$. Then $a\left(s_{j}\right)$ is an element in the fiber of $\tilde{E}$ over the first vertex of $s_{j}$. We let $\tau$ denote parallel translation back along $c$ to $m_{0}$. Then

$$
\alpha(c)=\tau\left(\sum_{j=1}^{n} \alpha\left(s_{j}\right)\right) .
$$

REMARK. The fiber of $\tilde{E}$ over the base point $m_{0}$ is $E$.

In order to make this calculation using a de Rham representative for $\alpha$ it is convenient to choose the lift $\hat{c}=\sum_{j=1}^{n} \hat{s}_{j}$ of $c$ to $\mathbf{H}$ starting at $z_{0}$. We observe that the value of $\operatorname{Sh} f$ on the oriented 1-simplex $\hat{s}_{i}=\left(v_{1}, v_{2}\right)$ is given by

$$
\left\langle\left\langle\operatorname{Sh} f,\left(v_{1}, v_{2}\right)\right\rangle\right\rangle=\left\langle\left\langle\omega_{f},\left(v_{1}, v_{2}\right)\right\rangle\right\rangle=\sum_{j=0}^{2 m}\left(\begin{array}{c}
2 m \\
j
\end{array}\right) \tilde{e}^{j}\left(v_{1}\right) \tilde{f}^{2 m-j}\left(v_{1}\right) \int_{\left(v_{1}, v_{2}\right)} z^{j} f(z) d z .
$$

Adding up over the simplices of $\hat{c}$ and applying the parallel translation we find the following formula for $r(f, \cdot)$, the crossed homomorphism on $\pi_{1}\left(M, m_{0}\right)$ associated to $\operatorname{Sh} f$.

THEOREM 1.1.

$$
r(f, \gamma)=\sum_{j=0}^{2 m}\left(\int_{z_{0}}^{\gamma z_{0}} z^{j} f(z) d z\right)\left(\begin{array}{c}
2 m \\
j
\end{array}\right) e^{j} f^{2 m-j}
$$

REMARK. $r(f, \cdot)$ is traditionally called the period polynomial of $f$.

Now assume $M$ has finite volume and restrict Sh to the cusp forms $S_{2 m+2}(\Gamma)$. We obtain

$$
\text { Sh: } S_{2 m+2}(\Gamma) \rightarrow H^{1}(\Gamma, E) .
$$

As a consequence of Theorem 1 of Shimura $[\mathbf{1 3}]$ we obtain the following theorem.

THEOREM 1.2. Sh is an injection.

We will need to know the image of Sh only in the compact quotient case. We assume then for the rest of this section that $M$ is compact. By the Dolbeault theorem (see Griffiths and Harris $[6]$ ) we have

$$
H^{0}(M, \mathcal{K} \otimes \tilde{E})=H^{1,0}(M, \tilde{E}) .
$$


We recall that $H^{1,0}(M, \tilde{E})$ consists of those de Rham cohomology classes that may be represented by closed forms of type $(1,0)$. We recall also that a 1 -form on $M$ with values in a complex vector bundle $E$ is said to have type $(1,0)$ if for every tangent vector $v$ we have $\omega(J v)=i \omega(v)$. Here $J$ is the almost complex structure on $T(M)$.

In this case by Matsushima and Murakami [10] we have a Hodge decomposition:

$$
H^{1}(M, \tilde{E}) \cong H^{1,0}(M, \tilde{E}) \oplus H^{0,1}(M, \tilde{E})
$$

Moreover, we may refine the above theorem (always assuming $M$ is compact).

THEOREM 1.2 (bis). Sh is an isomorphism onto $H^{1,0}(M, \tilde{E})$.

2. Eichler-Shimura period integrals. In this section we prove the formula stated in the introduction relating the Kronecker pairing of Sh $f$ and decomposable cycles to Eichler-Shimura periods of $f$ over closed geodesics. As a consequence we deduce a sufficient condition for a collection of hyperbolic Poincaré series to span the cusp forms.

LEMMA 2.1. If $\gamma \in \Gamma$ and $\gamma \neq \pm 1$ then $\gamma$ has a 1-dimensional space of invariants in $E^{*}$.

PROOF. The question of the dimension of the space of invariants for $\gamma$ depends only on the conjugacy class of $\gamma$ in $S L_{2}(\mathbf{R})$. Hence $\gamma$ may be put in one of two standard forms depending upon whether it is unipotent or semisimple. For these standard forms the lemma is obvious.

In what follows we will need to choose a nonzero element $\xi_{\gamma}$ in $E^{*}$ which is invariant under $\gamma$ in $\Gamma$. If $\gamma=\left[\begin{array}{ll}a & b \\ c & d\end{array}\right]$ then we define $s_{\gamma}$ in $S^{2} V^{*}$ and $\xi_{\gamma}$ in $E^{*}$ following S. Katok [8] by

$$
\begin{aligned}
& s_{\gamma}=c u^{2}+(d-a) u v-b v^{2}, \\
& \xi_{\gamma}=\left(c u^{2}+(d-a) u v-b v^{2}\right)^{m} .
\end{aligned}
$$

It is important to observe that $\xi_{\gamma} \in E_{\mathbf{R}}^{*}$.

We will later need to know how $s_{\gamma}$ and $\xi_{\gamma}$ transform as functions of $\gamma$ under $G L_{2}(\mathbf{R})$. We need only observe the following formula for $s_{\gamma}$. Let $p$ : End $V \rightarrow \mathfrak{g}$ be the projection on the trace zero matrices, so for $A \in$ End $V$ we have

$$
p(A)=A-\frac{1}{2}(\operatorname{tr} A) I .
$$

Let $B: \mathfrak{g} \rightarrow S^{2} V^{*}$ be the isomorphism given by the form (, ) (see $\S 1$ ). We then have the following formula

$$
s_{\gamma}=-B(p(\gamma))
$$

We find the following transformation law for $g \in G L_{2}(\mathbf{R})$.

LEMMA 2.2. $s_{g \gamma g^{-1}}=(\operatorname{det} g) \rho(g) s_{\gamma}$.

In what follows it will be more convenient to replace the Eilenberg-Mac Lane 1-cycle $\gamma \otimes \xi_{\gamma}$ with a simplicial 1-cycle with coefficients. Given $\gamma \in \Gamma$ we let $\alpha$ be the image under $\pi$ of any simplicial path joining $z_{0}$ to $\gamma z_{0}$. The invariant $\xi_{\gamma}$ will give rise, by parallel translation of $\xi_{\gamma}$ around $\alpha$, to a parallel section $\sigma_{\gamma}$ of $\tilde{E}^{*}$ restricted to $\alpha$. As in Johnson and Millson [7, §4], we can form a cycle with 
local coefficients denoted $\alpha \otimes \sigma_{\gamma}$. The class of $\alpha \otimes \sigma_{\gamma}$ in $H_{1}\left(M, \tilde{E}^{*}\right)$ will not be changed by a free homotopy of $\alpha$. Hence we may replace $\alpha$ by a closed geodesic if we so desire. We will denote the class of $\alpha \otimes \sigma_{\gamma}$ in simplicial homology with local coefficients by $[\gamma] \otimes \sigma_{\gamma}$.

We can consider periods over the previous cycles. If $\omega$ is a closed 1 -form on $M$ with values in $\tilde{E}$ we may restrict $\omega$ to $\alpha$ and pair with $\sigma_{\gamma}$ to obtain a closed 1 -form with scalar coefficients $\left\langle\omega, \sigma_{\gamma}\right\rangle$ on $\alpha$. We may then integrate $\left\langle\omega, \sigma_{\gamma}\right\rangle$ along $\alpha$ to get a period. In this way we may interpet the Kronecker pairing

$$
H^{1}(\Gamma, E) \otimes H_{1}\left(\Gamma, E^{*}\right) \rightarrow \mathbf{C}
$$

as obtained by taking periods of forms over simplicial cycles.

We now wish to consider the Kronecker pairing $\left\langle\left\langle\operatorname{Sh} f,[\gamma] \otimes \sigma_{\gamma}\right\rangle\right\rangle$ for $f \in S_{2 m+2}(\Gamma)$. We recall that we are regarding Sh $f$ as a $(1,0)$ form on $M$ with values in $\tilde{E}$ so the previous discussion applies. We have seen that the invariant corresponding to $\gamma$ is $\xi_{\gamma}=\left(c u^{2}+(d-a) u v-b v^{2}\right)^{m}$ where $\gamma=\left[\begin{array}{ll}a & b \\ c & d\end{array}\right]$. Using Lemma 1.5 we find

$$
\left\langle\operatorname{Sh} f(z), \sigma_{\gamma}\right\rangle=f(z)\left(c z^{2}+(d-a) z-b\right)^{m} d z .
$$

Integrating and observing that the integral is independent of the choice of the initial point $z_{0}$ and the path from $z_{0}$ to $\gamma z_{0}$, we obtain one of the main results of this paper.

THEOREM 2.1. For any point $z_{0} \in \mathbf{H}$ we have

$$
\left\langle\left\langle\operatorname{Sh} f(z),[\gamma] \otimes \sigma_{\gamma}\right\rangle\right\rangle=\int_{z_{0}}^{\gamma z_{0}} f(z)\left(c z^{2}+(d-a) z-b\right)^{m} d z
$$

where the integral is over any path joining $z_{0}$ to $\gamma z_{0}$.

COROLlaRY 1. If $\gamma$ is an elliptic element and $f$ is any element of $H^{0}\left(M, K^{m+1}\right)$ then

$$
\left\langle\left\langle\operatorname{Sh} f(z),[\gamma] \otimes \sigma_{\gamma}\right\rangle\right\rangle=0 .
$$

Proof. Choose $z_{0}$ to be the fixed point of $\gamma$.

COROLlaRY 2. If $\gamma$ is a parabolic element and $f \in S_{2 m+2}$ then

$$
\left\langle\left\langle\operatorname{Sh} f(z),[\gamma] \otimes \sigma_{\gamma}\right\rangle\right\rangle=0 .
$$

PROOF. Let $z_{0}$ tend toward the fixed point of $\gamma$.

COROLLARY 3. We have the following formula relating period polynomials and Eichler-Shimura periods:

$$
\left\langle r(f, \gamma), \xi_{\gamma}\right\rangle=\int_{z_{0}} \gamma z_{0} f(z)\left(c z^{2}+(d-a) z-b\right)^{m} d z .
$$

ProOF. We have seen that $r(f, \cdot)$ is an Eilenberg-Mac Lane 1-cocycle with values in $E$ representing $\operatorname{Sh} f$. Since the de Rham isomorphism is compatible with Kronecker pairings we have

$$
\left\langle\left\langle\operatorname{Sh} f,[\gamma] \otimes \sigma_{\gamma}\right\rangle\right\rangle=\left\langle\left\langle r(f, \cdot), \gamma \otimes \xi_{\gamma}\right\rangle\right\rangle .
$$

By definition of the Kronecker pairing of Eilenberg-Mac Lane cocyles and cycles we have

$$
\left\langle\left\langle r(f, \cdot), \gamma \otimes \xi_{\gamma}\right\rangle\right\rangle=\left\langle r(f, \gamma), \xi_{\gamma}\right\rangle \text {. }
$$


REMARK. From Corollary 3 we see that Eichler-Shimura period over the cycle $\gamma \otimes \xi_{\gamma}$ does not determine the period polynomial of $f$ evaluated at $\gamma$.

As another consequence of Theorem 2.1 we obtain the following theorem.

THEOREM 2.2. Let $\mathcal{W}$ be a (not necessarily proper) subset of $\Gamma$. Suppose the set of decomposable cycles $\Sigma=\left\{w \otimes \xi_{w}: w \in \mathcal{W}\right\}$ spans $H_{1}\left(\Gamma, E^{*}\right)$ as a complex vector space. Then the set of hyperbolic Poincaré series $\Theta=\left\{\Theta_{w}: w \in \mathcal{W}\right\}$ spans $S_{2 m+2}(\Gamma)$.

ProOF. Let $f \in S_{2 m+2}(\Gamma)$. Suppose $f$ is orthogonal to the span of $\Theta$. Then all Eichler-Shimura periods of $f$ over the hyperbolic cycles in $\Sigma$ vanish by the defining property of $\Theta_{w}$, that is

$$
\left(\left(f, \Theta_{w}\right)\right)_{2 m+2}=\int_{z_{0}}^{\gamma z_{0}} f(z)\left(c z^{2}+(d-a) z-b\right)^{m} d z
$$

where $((,))_{2 m+2}$ is the Petersson inner product on $S_{2 m+2}(\Gamma)$. Hence, by Theorem 2.1, Sh $f$ annihilates the hyperbolic cycles in $\Sigma$. But since $f$ is cuspidal Sh $f$ also annihilates the elliptic and parabolic cycles. But $\Sigma$ spans $H_{1}\left(\Gamma, E^{*}\right)$. Since the Kronecker pairing is perfect we find that $\operatorname{Sh} f=0$ and consequently $f=0$.

REMARKS. 1. The converse is false. Indeed, choose $\Sigma$ so that the homology classes of the elements of $\Sigma$ are a basis for a Lagrangian subspace of $H_{1}(\Gamma, \mathbf{R})$. Then $\Theta$ is a basis for $S_{2}(\Gamma)$, see Theorem 4.1 of Kudla and Millson [9]. But of course $\Sigma$ does not span $H_{1}(\Gamma, \mathbf{C})$.

2. Since $w \otimes \xi_{w}$ is a real class we see that the real span of $\Sigma$ is $H_{1}\left(\Gamma, E_{\mathbf{R}}^{*}\right)$ if and only if the complex span of $\Sigma$ is $H_{1}\left(\Gamma, E^{*}\right)$. We shall see in Chapter 4 that $\Theta$ spans $S_{2 m+2}(\Gamma)$ as a real vector space if and only if $\Sigma$ spans $H_{1}\left(\Gamma, E_{\mathbf{R}}^{*}\right)$.

3. Finally we remark that Theorem 2.2 is true whenever Sh is injective.

3. Hermitian structures on cohomology. From now on we assume $M$ is compact. We study Hermitian structures on $H^{1}(M, \tilde{E})$ obtained by its representation as the de Rham cohomology of forms with values in $\tilde{E}$. We denote the space of such forms by $A^{1}(M, \tilde{E})$. We observe that the invariant symmetric bilinear form on $E$ (and hence on $\tilde{E}$ ) when combined with the exterior product of forms gives us a skew-symmetric product, to be denoted $\wedge$,

$$
H^{1}(M, \tilde{E}) \otimes H^{1}(M, \tilde{E}) \rightarrow H^{2}(M, \mathbf{C})
$$

Since the latter group is isomorphic to $\mathbf{C}$ we obtain a complex bilinear skewsymmetric pairing on $H^{1}(M, \tilde{E})$ to be denoted [[ , ]] by evaluating on the fundamental class

$$
[[\eta, \tau]]=\int_{M} \eta \wedge \tau
$$

The form [[, ]] is real; that is, if $\eta$ and $\tau$ takes values in $\tilde{E}_{\mathbf{R}}$ then $[[\eta, \tau]]$ is real. By Poincaré duality $[[]$,$] is nonsingular.$

Definition. A closed form $\omega$ with values in $\tilde{E}$ is said to be Poincaré dual to $[\gamma] \otimes \sigma_{\gamma}$ if for every closed form $\eta$ with values in $\tilde{E}$ we have

$$
[[\eta, \omega]]=\left\langle\left\langle\eta,[\gamma] \otimes \sigma_{\gamma}\right\rangle\right\rangle .
$$

We will denote this class by $\operatorname{PD}\left([\gamma] \otimes \sigma_{\gamma}\right)$. 
We now construct a positive definite Hermitian inner product on $\tilde{E}$. We first observe that $V$ has an admissible inner product (see Borel and Wallach [2, p. 47] for the definition of admissible) to be denoted $(,)^{\prime}$ and defined by

$$
\left(v_{1}, v_{2}\right)^{\prime}=\left(J_{0} v_{1}, v_{2}\right), \quad \text { where } v_{1}, v_{2} \in V \text { and } J_{0}=\frac{1}{2}\left[\begin{array}{rr}
0 & 1 \\
-1 & 0
\end{array}\right] \text {. }
$$

Hence we obtain admissible inner products (again to be denoted $(,)^{\prime}$ ) on $S^{m} V$ for all $m$. We have the formula for $x, y \in V$ :

$$
\left(x^{m}, y^{m}\right)^{\prime}=\left(J_{0} x, y\right)^{m} \text {. }
$$

The following lemma relating the forms $(,)^{\prime}$ and $($,$) on L_{-} \subset E$ is immediate (for the definition of $L_{-}$see $\S 1$ ).

LEMMA 3.1. If $x$ and $y$ are in $L_{-}$then $(x, y)^{\prime}=(-1)^{m}(x, y)$.

REMARK. The previous formulas remain true by $G$-invariance for $x, y \in \mathcal{L}_{-}$and the $G$-invariant extensions of the forms $($,$) and (,)^{\prime}$ to $\tilde{E}$ which are again denoted $(,)^{\prime}$ and $($,$) .$

We can now construct a positive definite Hermitian inner product on $H^{1}(M, \tilde{E})$. We observe that $A^{1}(M, \tilde{E})$ has an almost complex structure $J$ given by

$$
J \omega(v)=-\omega(J v) .
$$

Here $J$ is the invariant almost complex structure on $T M$. An easy calculation shows that $J$ agrees with the operator $* \otimes 1$ where $*$ is the Hodge star on scalar-valued forms. We define the Hodge star $*$ on bundle-valued 1 -forms by requiring $*=J$. We have

$$
* \eta \wedge * \tau=\eta \wedge \tau \text {. }
$$

The action of $J$ on forms commutes with $d$ and we obtain an almost complex structure, again denoted $J$, on $H^{1}(M, \tilde{E})$. We may then define a positive definite Hermitian form to be denoted $(()$,$) on A^{1}(M, \tilde{E})$ by

$$
((\eta, \tau))=\int_{M} \eta \wedge^{\prime} \overline{* \tau}
$$

Here by $\wedge^{\prime}$ we mean the exterior product formed by using the form $(,)^{\prime}$ on the coefficients. The form $(()$,$) induces a Hermitian structure on H^{1}(M, \tilde{E})$.

We may use the form $(()$,$) to construct a Laplacian \Delta$ and obtain harmonic representatives of cohomology classes. There is a unique harmonic form satisfying the defining equation for $\operatorname{PD}\left([\gamma] \otimes \sigma_{\gamma}\right)$. We will call this form the Poincaré dual of $[\gamma] \otimes \sigma_{\gamma}$.

We recall that $\eta \in A^{1}(M, \tilde{E})$ is said to be holomorphic if it is of type $(1,0)$ and $\bar{\partial} \eta=0$ where $\bar{\partial} \eta$ is the type $(0,1)$ projection of $d \eta$. Also if $\eta$ has type $(1,0)$ it is holomorphic if and only if it is harmonic. By the theorem of MatsushimaMurakami, see Murakami [11], Lemma 5.4, a harmonic $(1,0)$ form takes values in $\mathcal{L}_{-}$, the lowest weight bundle. Hence we find the following simple relationship between the $(()$,$) and [[]$,$] on holomorphic bundle-valued 1-forms or equivalently$ on $H^{1,0}(M, \tilde{E})$.

The following lemma is an immediate consequence of Lemma 3.1. 
LEMMA 3.2. If $\omega_{1}$ and $\omega_{2}$ are holomorphic $\tilde{E}$-valued 1 -forms we have

$$
\left(\left(\omega_{1}, \omega_{2}\right)\right)=(-1)^{m}\left[\left[\omega_{1}, \overline{{ }^{*} \omega_{2}}\right]\right] .
$$

We also wish to compare the pull-back of $(()$,$) under Sh with the Petersson$ inner product $((,))_{2 m+2}$ on $S_{2 m+2}(\Gamma)$.

LEMMA 3.3 .

$$
((f, g))_{2 m+2}=\frac{1}{2^{2 m+1}}((\operatorname{Sh} f, \operatorname{Sh} g)) .
$$

ProOF. From formula (6) in Shimura $[\mathbf{1 3}]$ we have

$$
((f, g))_{2 m+2}=-\frac{1}{(2 i)^{2 m+1}}[[\operatorname{Sh} f, \overline{\operatorname{Sh} g}]] .
$$

The lemma follows from this and the fact that Sh $g$ has type $(1,0)$ and consequently ${ }^{*}$ Sh $g=-i$ Sh $g$.

In what follows we will need the action of certain elements of $G L_{2}(\mathbf{R})$ of determinant -1 on the form $(()$,$) . Suppose \varepsilon$ is such an element which normalizes $\Gamma$. Then we can make $\varepsilon$ operate on $H^{1}(\Gamma, E)$ by the formula

$$
\varepsilon c(\gamma)=\rho(\varepsilon) c\left(\varepsilon \gamma \varepsilon^{-1}\right),
$$

for $c$ a representing cocycle. Here we have used $\rho(\varepsilon)$ to denote the action of $\varepsilon$ on $E$.

We now examine the corresponding action of $\varepsilon$ on $A^{1}(M, \tilde{E})$. We first observe that if $\varepsilon=\left[\begin{array}{ll}a & b \\ c & d\end{array}\right]$ then $\varepsilon$ acts on $\mathbf{H}$ by the formula

$$
\varepsilon z=(a \bar{z}+b) /(c \bar{z}+d) .
$$

Since $\varepsilon$ normalizes $\Gamma$ this action descents to $M$. We denote the action on $M$ again by $\varepsilon$. We also obtain an action to be denoted $\rho(\varepsilon)$ on $\tilde{E}$ as follows:

$$
\rho(\varepsilon)[z, v]=[\varepsilon z, \rho(\varepsilon) v] .
$$

Here $[z, v]$ denotes the equivalence class of $(z, v)$ in $\mathbf{H} \times E$.

Then $\varepsilon$ acts on $\mathscr{A}^{1}(M, \tilde{E})$ by the formula

$$
\varepsilon \omega=\rho(\varepsilon) \circ \varepsilon^{*} \omega=\rho(\varepsilon) \circ \omega \circ d \varepsilon .
$$

Let $\eta$ and $\tau$ be elements of $\mathcal{A}^{1}(M, \tilde{E})$. An easy calculation yields

$$
\varepsilon \eta \wedge \varepsilon \tau=\varepsilon^{*} \eta \wedge \varepsilon^{*} \tau=\varepsilon^{*}(\eta \wedge \tau) .
$$

Then we have the following lemma.

LEMMA 3.4. (i) $[[\varepsilon \eta, \varepsilon \tau]]=-[[\eta, \tau]]$,

(ii) $((\varepsilon \eta, \varepsilon \tau))=((\eta, \tau))$.

ProOF. We have $\varepsilon \eta \wedge \varepsilon \tau=\varepsilon^{*}(\eta \wedge \tau)$. Hence

$$
[[\varepsilon \eta, \varepsilon \tau]]=\int_{M} \varepsilon^{*}(\eta \wedge \tau)=\int_{\varepsilon * M} \eta \wedge \tau=-\int_{M} \eta \wedge \tau=-[[\eta, \tau]] .
$$

We also have $\varepsilon \eta \wedge^{\prime} \varepsilon \tau=\varepsilon^{*}\left(\eta \wedge^{\prime} \tau\right)$. Noting that $\varepsilon$ anticommutes with $*$ we have

$$
((\varepsilon \eta, \varepsilon \tau))=\int_{M} \varepsilon \eta \wedge^{\prime} \overline{{ }^{*} \varepsilon \tau}=-\int_{M} \varepsilon \eta \wedge^{\prime} \varepsilon^{*} \tau=-\int_{M} \varepsilon^{*}\left(\eta \wedge^{\prime} \bar{*} \tau\right)=((\eta, \tau)) .
$$

With this the lemma is proved. 
4. The Poincaré dual of a hyperbolic decomposable cycle. In this section we give the precise relationship between $\operatorname{Sh} \Theta_{\gamma}$ and $\operatorname{PD}\left([\gamma] \otimes \sigma_{\gamma}\right)$. We use this to prove the equivalence of the problem of finding a collection of hyperbolic Poincaré series which span $S_{2 m+2}(\Gamma)$ as a real vector space and a collection of decomposable cycles which span $H_{1}\left(\Gamma, E_{\mathbf{R}}^{*}\right)$. We can then apply results of S. Katok $[\mathbf{8}]$ and deduce that $H_{1}\left(\Gamma, E_{\mathbf{R}}^{*}\right)$ is spanned by decomposable cycles.

We begin with the following theorem.

THEOREM 4.1.

$$
\operatorname{Im} \operatorname{Sh} \Theta_{\gamma}=(-1)^{m} 2^{2 m} \mathrm{PD}\left([\gamma] \otimes \sigma_{\gamma}\right)
$$

The proof of this theorem follows from the ensuing discussion.

By Hodge theory, there exists a unique harmonic 1-form $\Omega_{\gamma}$ on $M$ with values in $\tilde{E}$ such that if $\eta$ is any closed 1 -form with values in $\tilde{E}$ we have

$$
\left[\left[\eta, \Omega_{\gamma}\right]\right]=\int_{M} \eta \wedge \Omega_{\gamma}=\left\langle\left\langle\eta,[\gamma] \otimes \sigma_{\gamma}\right\rangle\right\rangle
$$

that is $\Omega_{\gamma}=\operatorname{PD}\left([\gamma] \otimes \sigma_{\gamma}\right)$.

We put $\Phi_{\gamma}=-J \Omega_{\gamma}=-{ }^{*} \Omega_{\gamma}$ and we define

$$
\Xi_{\gamma}=\frac{1}{2}\left[\Phi_{\gamma}+i \Omega_{\gamma}\right]
$$

We observe that $\Omega_{\gamma}$ and $\Phi_{\gamma}$ are real. Therefore, $\operatorname{Im} \Xi_{\gamma}=\frac{1}{2} \Omega_{\gamma}$. We observe that $\Xi_{\gamma}$ is a holomorphic 1 -form (that is, a harmonic 1-form of type $(1,0)$ ) with values in $\tilde{E}$.

We define a holomorphic 1-form $\Xi$ with values in $\tilde{E}$ to be associated to the cycle $[\gamma] \otimes \sigma_{\gamma}$ as above if for every holomorphic 1 -form $\eta$ with values in $\tilde{E}$ we have $((\eta, \Xi))=\left\langle\left\langle\eta,[\gamma] \otimes \sigma_{\gamma}\right\rangle\right\rangle$. Here $(()$,$) is the Hermitian form on bundle-valued$ 1 -forms described in the previous section. We recall (Lemma 3.3) that $\mathrm{Sh}$ is an isometry from $S_{2 m+2}$ to the holomorphic 1-forms equipped with the Hermitian form $2^{-(2 m+1)}(()$,$) .$

LEMMA 4.1. $(-1)^{m} \Xi_{\gamma}$ is the bundle-valued holomorphic 1-form associated to $[\gamma] \otimes \sigma_{\gamma}$.

PROOF. Let $\eta$ be a holomorphic 1-form. Then by Lemma 3.2:

$$
\left(\left(\eta, \Xi_{\gamma}\right)\right)=(-1)^{m}\left[\left[\eta,{ }^{*} \Xi_{\gamma}\right]\right]=(-1)^{m} \frac{1}{2}\left\{\left[\left[\eta,{ }^{*} \Phi_{\gamma}\right]\right]-i\left[\left[\eta,{ }^{*} \Omega_{\gamma}\right]\right]\right\} .
$$

But $\eta$ has type $(1,0)$; hence ${ }^{*} \eta=-i \eta$. We obtain

$$
\left[\left[\eta,{ }^{*} \Omega_{\gamma}\right]\right]=\int_{M} \eta \wedge^{*} \Omega_{\gamma}=-\int_{M}{ }^{*} \eta \wedge \Omega_{\gamma}=i \int_{M} \eta \wedge \Omega_{\gamma}=i\left[\left[\eta, \Omega_{\gamma}\right]\right] .
$$

Also by definition ${ }^{*} \Phi_{\gamma}=\Omega_{\gamma}$ and we obtain

$$
\left(\left(\eta, \Xi_{\gamma}\right)\right)=(-1)^{m} \frac{1}{2}\left\{\left[\left[\eta, \Omega_{\gamma}\right]\right]+i\left[\left[\eta, \Omega_{\gamma}\right]\right]\right\}=(-1)^{m}\left\langle\left\langle\eta,[\gamma] \otimes \sigma_{\gamma}\right\rangle\right\rangle .
$$

With this the lemma is proved.

COROLlaRY. $\Xi_{\gamma}=(-1)^{m} 2^{-(2 m+1)} \mathrm{Sh} \Theta_{\gamma}$.

PROOF. From Lemma 3.3 we have for any bundle-valued holomorphic 1-form $\eta$

$$
2^{-(2 m+1)}\left(\left(\eta, \operatorname{Sh} \Theta_{\gamma}\right)\right)=\left\langle\left\langle\eta,[\gamma] \otimes \sigma_{\gamma}\right\rangle\right\rangle
$$

and the corollary follows.

But since $\operatorname{PD}\left([\gamma] \otimes \sigma_{\gamma}\right)=2 \operatorname{Im} \Xi_{\gamma}$ we have now proved Theorem 4.1. 
THEOREM 4.2. Let $\mathcal{W}$ be a (not necessary proper) subset of $\Gamma$. Then the set of decomposable cycles $\Sigma=\left\{w \otimes \xi_{w}: w \in \mathcal{W}\right\}$ spans $H_{1}\left(\Gamma, E_{\mathbf{R}}^{*}\right)$ if and only if $\Theta=\left\{\Theta_{w}: w \in \mathcal{W}\right\}$ spans $S_{2 m+2}(\Gamma)$ as a real vector space.

Proof. Suppose that $\Sigma$ spans $H_{1}\left(\Gamma, E_{\mathbf{R}}^{*}\right)$. Then the complex span of $\Sigma$ is $H_{1}\left(\Gamma, E^{*}\right)$ and we are done by Theorem 2.2 .

Conversely suppose $\Theta$ spans $S_{2 m+2}(\Gamma)$ as a real vector space. The map $f \rightarrow$ $\operatorname{Im} f$ is easily seen to be an isomorphism of the real vector spaces $S_{2 m+2}(\Gamma)$ and $H^{1}\left(\Gamma, E_{\mathbf{R}}\right)$. Then the image of $\Theta$ under this isomorphism, $\left\{\Omega_{w}: w \in \mathcal{W}\right\}$ spans $H^{1}\left(\Gamma, E_{\mathbf{R}}\right)$. By Theorem 4.1 the classes $\left\{\operatorname{PD}\left(w \otimes \xi_{w}\right): w \in \mathcal{W}\right\}$ span $H^{1}\left(\Gamma, E_{\mathbf{R}}\right)$ and we are done since $\mathrm{PD}$ is an isomorphism.

THEOREM 4.3. The set of all hyperbolic Poincaré series spans $S_{2 m+2}(\Gamma)$ as a real vector space in case $\Gamma$ is a discrete subgroup of $S L_{2}(\mathbf{R})$ which is normalized by $\varepsilon=\left[\begin{array}{rr}-1 & 0 \\ 0 & 1\end{array}\right]$.

PROOF. The result for arithmetic subgroups is contained in S. Katok [8]. The argument for a general $\Gamma$ is very similar to that, but for completeness sake we will give it.

Let $\gamma^{\prime}=\varepsilon \gamma \varepsilon$ and

$$
\Theta_{m+1, \gamma}^{+}=\frac{1}{2}\left(\Theta_{m+1, \gamma}+\Theta_{m+1, \gamma^{\prime}}\right), \quad \Theta_{m+1, \gamma}^{-}=(1 / 2 i)\left(\Theta_{m+1, \gamma}-\Theta_{m+1, \gamma^{\prime}}\right) .
$$

Let $S_{\mathbf{R}}^{ \pm}(\Gamma)$ be the $\mathbf{R}$-span of $\left\{\Theta_{m+1, \gamma}^{ \pm}\right\}$and $S_{\mathbf{R}}^{0}(\Gamma)$ be the $\mathbf{R}$-span of $\left\{\Theta_{m+1, \gamma}\right\}$. For any two hyperbolic elements $\gamma_{0}, \gamma_{1} \in \Gamma$ we have

$$
\left(\left(\Theta_{m+1, \gamma_{0}}^{+}, \Theta_{m+1, \gamma_{1}}^{-}\right)\right)_{2 m+2} \in \mathbf{R} \quad \text { and } \quad\left(\left(\Theta_{m+1, \gamma_{0}}^{-}, \Theta_{m+1, \gamma_{1}}^{-}\right)\right)_{2 m+2} \in \mathbf{R} .
$$

Then using Theorem 1(ii) of $[8]$ we have

$$
\operatorname{dim}_{\mathbf{R}} S_{\mathbf{R}}^{+}(\Gamma)=\operatorname{dim}_{\mathbf{C}} S_{2 m+2}(\Gamma) \text { and } S_{\mathbf{R}}^{+} \cap i S_{\mathbf{R}}^{-}=\{0\},
$$

which implies $S_{\mathbf{R}}^{0}(\Gamma)=S_{\mathbf{R}}^{+} \oplus i S_{\mathbf{R}}^{-}$. This completes the proof.

As a consequence of this theorem and Theorem 4.2 we have the following theorem.

THEOREM 4.4. The set of all decomposable cycles $\left\{\gamma \otimes \xi_{\gamma}: \gamma\right.$ hyperbolic $\}$ spans $H_{1}\left(\Gamma, E_{\mathbf{R}}^{*}\right)$.

5. Rational structures on $S_{2 m+2}(\Gamma)$. We show how the existence of two natural rational structures $S_{2 m+2}^{+}(\Gamma)$ and $S_{2 m+2}^{-}(\Gamma)$ follows from the Shimura isomorphism. We recall that a rational structure on a complex vector space $V$ is a rational vector space $W$ and an isomorphism from $W \otimes_{\mathbf{Q}} \mathbf{C}$ to $V$.

We now assume that our cocompact group $\Gamma$ is arithmetic of the following type: $\Gamma$ is commensurable with the group of units of an order $O$ contained in a division quaternion algebra $B$, defined over $Q$, such that $B \otimes_{\mathbf{Q}} \mathbf{R}$ is isomorphic to the algebra of 2 by 2 real matrices $M_{2}(\mathbf{R})$.

LEMMA 5.1. There exists a $\Gamma$-invariant rational subspace $W_{2 m}$ of $S^{2 m} \mathbf{C}^{2}$ such that the natural map $H^{1}\left(\Gamma, W_{2 m}\right) \otimes_{\mathbf{Q}} \mathbf{C} \rightarrow H^{1}\left(\Gamma, S^{2 m} \mathbf{C}^{2}\right)$ is an isomorphism.

PROOF. Let $B^{0}$ be the traceless quaternions contained in the order 0 . Then $B^{0}$ induces a $\Gamma$-invariant rational subspace on $S^{2} \mathbf{C}^{2}$ denoted by $L$. The lattice $L$ induces a $\Gamma$-invariant rational structure $S^{m} L$ on $S^{m}\left(S^{2} \mathbf{C}^{2}\right)$. We wish to prove 
that $S^{2 m} \mathbf{C}^{2}$ has a $\Gamma$-invariant rational structure. For this it is sufficient to show that the above rational structure induces a rational structure on the kernel of the projection

$$
p: S^{m}\left(S^{2} \mathbf{C}^{2}\right) \rightarrow S^{2 m} \mathbf{C}^{2} .
$$

As indicated in $\S 1$, for $k \geq 2$ the space $S^{k}\left(S^{2} \mathbf{C}^{2}\right)$ may be realized as the space of all homogeneous polynomials of degree $k$ in three variables, coordinates with respect to the basis of the lattice $W$ in $S^{2} \mathbf{C}^{2}$, which are quadratic forms in two variables. The projection

$$
p_{k}: S^{k}\left(S^{2} \mathbf{C}^{2}\right) \rightarrow S^{2 k} \mathbf{C}^{2}
$$

may then be realized by substitution: it projects the space $S^{k}\left(S^{2} \mathbf{C}^{2}\right)$ onto $S^{2 k} \mathbf{C}^{2}$, the space of all homogeneous polynomials of degree $2 k$ in two variables. For $f_{i} \in$ $S^{i}\left(S^{2} \mathbf{C}^{2}\right)$ and $f_{j} \in S^{j}\left(S^{2} \mathbf{C}^{2}\right)$ we have $p_{i}\left(f_{i}\right) \cdot p_{j}\left(f_{j}\right)=p_{i+j}\left(f_{i} \cdot f_{j}\right)$ where $\cdot$ is the product of polynomials. The homomorphism

$$
p_{2}: S^{2}\left(S^{2} \mathbf{C}^{2}\right) \rightarrow S^{4} \mathbf{C}^{2}
$$

intertwines with the action of $P S L_{2}(\mathbf{R})$. Therefore $\operatorname{Ker} p_{2}$ is an invariant subspace for $P S L_{2}(\mathbf{R})$. Computing dimensions we get $\operatorname{dim} S^{2}\left(S^{2} \mathbf{C}^{2}\right)=6, \operatorname{dim} S^{4} \mathbf{C}^{2}=5$ and therefore $\operatorname{dim} \operatorname{Ker} p_{2}=1$. But the only 1-dimensional representation of $P S L_{2}(\mathbf{R})$ is trivial, so $\operatorname{Ker} p_{2}$ is a line fixed by $P S L_{2}(\mathbf{R})$, and therefore by $\Gamma$. Consider the basis in $S^{2}\left(S^{2} \mathbf{C}^{2}\right)$ which belongs to the $\Gamma$-invariant subspace $S^{2} L$. In this basis the group $\Gamma$ has rational matrices. $\operatorname{Ker} p_{2}$ is defined by the vector of $S^{2}\left(S^{2} \mathbf{C}^{2}\right)$ of eigenvalue 1 for all matrices from $\Gamma$ which then has rational coordinates and therefore belongs to $S^{2} L$. We denote this vector by $\alpha$. It is easy to see that

$$
\operatorname{dim} S^{m}\left(S^{2} \mathbf{C}^{2}\right)=\operatorname{dim} S^{m-1}\left(S^{2} \mathbf{C}^{2}\right)+m+1
$$

and therefore

$$
\operatorname{dim} S^{m}\left(S^{2} \mathbf{C}^{2}\right)=\operatorname{dim} S^{m-2}\left(S^{2} \mathbf{C}^{2}\right)+2 m+1=\operatorname{dim} S^{m-2}\left(S^{2} \mathbf{C}^{2}\right)+\operatorname{dim} S^{2 m} \mathbf{C}^{2}
$$

and

$$
\operatorname{dim} \operatorname{Ker} p_{m}=\operatorname{dim} S^{m-2}\left(S^{2} \mathbf{C}^{2}\right) .
$$

Since $p_{2}(\alpha)=0$ for any $f_{m-2} \in S^{m-2}\left(S^{2} \mathbf{C}^{2}\right)$ we have $p_{m}\left(f_{m-2} \cdot \alpha\right)=0$, that is $S^{m-2}\left(S^{2} \mathbf{C}^{2}\right) \cdot \alpha \subset \operatorname{Ker} p_{m}$. Therefore $S^{m-2}\left(S^{2} \mathbf{C}^{2}\right) \cdot \alpha=\operatorname{Ker} p_{m}$, and $S^{m-2} W \cdot \alpha \subset$ $S^{m} L$ gives a rational structure on $\operatorname{Ker} p_{m}=\operatorname{Ker} p$. With this we have proved the existence of the rational structure on $S^{2 m} \mathbf{C}^{2}$ to be denoted $W_{2 m}$. Since the tensoring over a field is exact the lemma is proved.

LEMMA 5.2. $\xi_{\gamma} \in W_{2 m}$ for all hyperbolic $\gamma \in \Gamma$.

PROOF. It follows from the argument of Lemma 5.1 that it is sufficient to show that $s_{\gamma} \in L$ for all hyperbolic $\gamma \in \Gamma$. We have seen in $\S 2$ that $p(\gamma)=\gamma-\frac{1}{2}(\operatorname{tr} \gamma) I=$ $\frac{1}{2}\left(\gamma-\gamma^{-1}\right) \in \mathfrak{g}$. But since $\gamma, \gamma^{-1} \in \mathcal{O}, p(\gamma)$ is in the rational subspace of $\mathfrak{g}$ generated by $B^{0}$, and hence $s_{\gamma}=-B(p(\gamma)) \in L$.

LEMMA 5.3. The form (, ) takes rational values on $W_{2 m}$.

PROOF. Since the projection $p$ intertwines with the action of $P S L_{2}(\mathbf{R})$, the group $\Gamma$ has rational matrices in the basis of $W_{2 m}$ induced by $p$. It follows immediately from irreducibility of $S^{2 m} \mathbf{C}^{2}$ that any $P S L_{2}(\mathbf{C})$-invariant symmetric bilinear form on $S^{2 m} \mathbf{C}^{2}$ is a multiple of $($,$) . By the Borel density theorem, Raghunathan$ 
[12, Chapter V], the same statement holds with $P S L_{2}(\mathbf{C})$ replaced by $\Gamma$. But since $\Gamma$ acts with rational entries we conclude that there exists a complex number $\lambda$ such that $\lambda($,$) takes rational values on W_{2 m}$. However, by Lemma 5.2 we have $\xi_{\gamma} \in W_{2 m}$ for $\gamma \in \Gamma$, and it is easily seen that $\left(\xi_{\gamma}, \xi_{\gamma}\right)$ is rational (see computations in $\S 7$ ). Consequently $\lambda$ is rational and may be chosen to be 1 . With this the lemma is proved.

In what follows $m$ will play no role and we will abbreviate $W_{2 m}$ by $W$. We now assume that $\Gamma$ is normalized by an orientation reversing isometry $\varepsilon$ of order 2 . We assume that $\varepsilon$ preserves $\mathcal{O} \otimes \mathbf{Q}$ in its action on $B^{0}$. Then $\varepsilon$ preserves $W$. Hence, the action of $\varepsilon$ on $H^{1}(\Gamma, E)$ discussed in the previous section preserves $H^{1}(\Gamma, W)$. We may decompose $H^{1}(\Gamma, W)$ into a direct sum of +1 and -1 eigenspaces for $\varepsilon$. We denote those spaces by $H^{1}(\Gamma, W)^{+}$and $H^{1}(\Gamma, W)^{-}$respectively. We let $N$ denote the dimension over $\mathbf{C}$ of $H^{1}(\Gamma, E)^{-}$of course; this is equal to the dimension of $H^{1}(\Gamma, W)$ over $\mathbf{Q}$. We have seen that the de Rham cohomology $H^{1}(M, \tilde{E})$ admits a nondegenerate skew-symmetric form. Hence $N$ is even. The corresponding symplectic structure on $H^{1}(\Gamma, E)$ may be described directly by the cup-product of 1-cycles followed by the evaluation on the fundamental class. We again denote it by $[[]$,$] . According to Lemma 5.3, the form [[]$,$] takes rational values on H^{1}(\Gamma, W)$.

LEMMA 5.4. $\operatorname{dim} H^{1}(\Gamma, W)^{+}=\operatorname{dim} H^{1}(\Gamma, W)^{-}=\frac{1}{2} N$.

Proof. By Lemma $3.4 \varepsilon$ changes the symplectic form [[ , ]] on $H^{1}(\Gamma, W)$ to its negative. Hence the two eigenspaces are totally isotropic. But since $H^{1}(\Gamma, W)$ is a direct sum of these two spaces each space must be Lagrangian.

Now let Sh: $S_{2 m+2}(\Gamma) \rightarrow H^{1}(\Gamma, E)$ be the Shimura map. We let $p_{+}$and $p_{-}$be the projections of $H^{1}(\Gamma, H)$ onto $H^{1}(\Gamma, E)^{+}$and $H^{1}(\Gamma, E)^{-}$respectively. From the universal coefficient theorem we have

$$
H^{1}(\Gamma, W)^{+} \otimes_{\mathbf{Q}} \mathbf{C} \cong H^{1}(\Gamma, E)^{+}, \quad H^{1}(\Gamma, W)^{-} \otimes_{\mathbf{Q}} \mathbf{C} \cong H^{1}(\Gamma, E)^{-} .
$$

Thus we will have found the desired rational structures if we prove the following lemma.

LEMMA 5.5. $p_{+} \circ \mathrm{Sh}$ and $p_{-} \circ \mathrm{Sh}$ are isomorphisms.

ProOF. By a dimension count it is sufficient to prove that the above maps are injective. This is in turn equivalent to the statements

(i) $\operatorname{Sh}\left(S_{2 m+2}(\Gamma)\right) \cap H^{1}(\Gamma, E)^{+}=\{0\}$,

(ii) $\operatorname{Sh}\left(S_{2 m+2}(\Gamma)\right) \cap H^{1}(\Gamma, E)^{-}=\{0\}$.

But we have seen that the image of Sh is contained in $H^{1,0}(\Gamma, E)$. Thus to complete the proof it is sufficient to check that $\varepsilon$ interchanges $H^{1,0}(\Gamma, E)$ and $H^{0,1}(\Gamma, E)$.

We have seen $\S 3$ that the induced action of $\varepsilon$ on $\AA^{1}(M, \tilde{E})$ is given by

$$
\varepsilon \omega(v)=\rho(\varepsilon) \omega(d \varepsilon(v)) .
$$

But the action of $\rho(\varepsilon)$ commutes with $J$ (they operate on opposite sides of $\omega$ ) and $d \varepsilon$ anticommutes with $J$. Hence $\varepsilon$ anticommutes with $J$ and interchanges the two eigenspaces of $J$. With this the lemma is proved.

Thus we obtain a rational structure on $S_{2 m+2}(\Gamma)$ considered as a real vector space:

$$
S_{2 m+2}^{0}(\Gamma)=\left\{f \in S_{2 m+2}(\Gamma): \operatorname{Im} \operatorname{Sh} f \in H^{1}(\Gamma, W)\right\}
$$


and two rational structures on $S_{2 m+2}(\Gamma)$ considered as a complex vector space:

$$
\begin{aligned}
& S_{2 m+2}^{+}(\Gamma)=\left\{f \in S_{2 m+2}(\Gamma): p_{+} \circ \operatorname{Sh} f \in H^{1}(\Gamma, W)^{+}\right\}, \\
& S_{2 m+2}^{-}(\Gamma)=\left\{f \in S_{2 m+2}(\Gamma): p_{-} \circ \operatorname{Sh} f \in H^{1}(\Gamma, W)^{-}\right\} .
\end{aligned}
$$

REMARK. The rational structure $S_{2 m+2}^{+}(\Gamma)$ may be described as those $f$ whose period polynomials $r(f, \cdot)$ satisfy

$$
r(f, \varepsilon \gamma \varepsilon)+\rho(\varepsilon) r(f, \gamma) \in W \quad \text { for all } \gamma \in \Gamma .
$$

Similarly the rational structure $S_{2 m+2}^{-}(\Gamma)$ may be described as those $f$ whose period polynomials $r(f, \cdot)$ satisfy

$$
r(f, \varepsilon \gamma \varepsilon)-\rho(\varepsilon) r(f, \gamma) \in W \quad \text { for all } \gamma \in \Gamma .
$$

In more concrete terms $f \in S_{2 m+2}^{+}(\Gamma)$ if and only if

$$
\begin{array}{ll}
\int_{z_{0}}^{\gamma z_{0}} z^{j} f(z) d z+\int_{z_{0}}^{\gamma^{\prime} z_{0}} z^{j} f(z) d z \in \mathbf{Q} & \text { for } j \text { even, } \\
\int_{z_{0}}^{\gamma z_{0}} z^{j} f(z) d z-\int_{z_{0}}^{\gamma^{\prime} z_{0}} z^{j} f(z) d z \in \mathbf{Q} & \text { for } j \text { odd. }
\end{array}
$$

6. Comparison with the rational structure coming from relative Poincaré series. In this section we relate the rational structures $S_{2 k}^{0}(\Gamma), S_{2 k}^{+}(\Gamma)$ and $S_{2 k}^{-}(\Gamma)$ with the rational structures constructed in S. Katok $[8]$ and denoted $S_{2 k}^{0}(\Gamma)$, $S_{2 k}^{+}(\Gamma)$ and $S_{2 k}^{-}(\Gamma)$ there. For this and the next section we switch from $m$ to $k-1$ to conform to $[8]$.

We prove the following theorem.

THEOREM 6.1. (i) $S_{2 k}^{0}(\Gamma)=S_{2 k}^{0}(\Gamma)$;

(ii) $S_{2 k}^{+}(\Gamma)=i S_{2 k}^{+}(\Gamma)$ and $S_{2 k}^{-}(\Gamma)=S_{2 k}^{-}(\Gamma)$ if $k$ is even, $S_{2 k}^{-}(\Gamma)=i S_{2 k}^{+}(\Gamma)$ and $S_{2 k}^{+}(\Gamma)=S_{2 k}^{-}(\Gamma)$ if $k$ is odd.

PROOF. We begin by recalling the definitions of the rational structures $S_{2 k}^{0}(\Gamma)$, $S_{2 k}^{+}(\Gamma)$ and $S_{2 k}^{-}(\Gamma)$. Let $\Theta_{k, \gamma}$ be the relative Poincaré series. The elements $\Theta_{k, \gamma}^{+}$ and $\Theta_{k, \gamma}^{-}$of $S_{2 k}(\Gamma)$ are defined as in $\S 4$, Theorem 4.3. It was proved in S. Katok [8] that the Q-span of $\left\{\Theta_{k, \gamma}, \gamma \in \Gamma\right\}$ was a rational structure $S_{2 k}^{0}(\Gamma)$ for $S_{2 k}(\Gamma)$ as a real vector space, the $\mathbf{Q}$-span of $\left\{\Theta_{k, \gamma}^{+}, \gamma \in \Gamma\right\}$ was a rational structure $S_{2 k}^{+}(\Gamma)$ and the Q-span of $\left\{\Theta_{k, \gamma}^{-}, \gamma \in \Gamma\right\}$ was a rational structure $S_{2 k}^{-}(\Gamma)$ for $S_{2 k}(\Gamma)$ as a complex vector space.

By Lemma 5.2 we have $\gamma \otimes \xi_{\gamma} \in H_{1}\left(\Gamma, W_{2 k-2}^{*}\right)$. Then

$$
\Omega_{\gamma}=\frac{1}{2} \operatorname{Im} \operatorname{Sh} \Theta_{\gamma}=(-1)^{m} 2^{2 m-1} \operatorname{PD}\left(\gamma \otimes \xi_{\gamma}\right) \in H^{1}\left(\Gamma, W_{2 k-2}\right) \text {, }
$$

and part (i) follows.

Since $p_{+} \circ$ Sh and $p_{-} \circ$ Sh are injective, part (ii) will follow if we can prove that for $k$ even

(i) $p_{+}\left(\operatorname{Sh}\left(S_{2 k}^{+}(\Gamma)\right)\right) \subset i H^{1}\left(\Gamma, W_{2 k-2}\right)^{+}$,

(ii) $p_{-}\left(\operatorname{Sh}\left(S_{2 k}^{-}(\Gamma)\right)\right) \subset H^{1}\left(\Gamma, W_{2 k-2}\right)^{-}$,

and for $k$ odd

(iii) $p_{-}\left(\operatorname{Sh}\left(S_{2 k}^{+}(\Gamma)\right)\right) \subset i H^{1}\left(\Gamma, W_{2 k-2}\right)^{-}$,

(iv) $p_{+}\left(\operatorname{Sh}\left(S_{2 k}^{-}(\Gamma)\right)\right) \subset H^{1}\left(\Gamma, W_{2 k-2}\right)^{+}$. 
Indeed since the two spaces in (i) have the same dimension they must coincide and consequently their inverse images under $p_{+} \circ$ Sh coincide. But these inverse images are $S_{2 k}^{+}(\Gamma)$ and $S_{2 k}^{+}(\Gamma)$ respectively. A similar argument applies to the spaces in (ii)-(iv).

LEMMA 6.1. (i) $\varepsilon \Omega_{\gamma}=(-1)^{k} \Omega_{\gamma^{\prime}}$,

(ii) $\varepsilon \Phi_{\gamma}=(-1)^{k+1} \Phi_{\gamma^{\prime}}$,

(iii) $\varepsilon \Xi_{\gamma}=(-1)^{k+1} \Xi_{\gamma^{\prime}}$.

PROOF. To prove the first formula we observe that Lemma 2.2 implies $\rho(\varepsilon) s_{\gamma}=$ $-s_{\gamma^{\prime}}$ and consequently $\rho(\varepsilon) s_{\gamma}^{k-1}=(-1)^{k-1} s_{\gamma^{\prime}}^{k-1}$. The first formula now follows from the naturality of Poincaré duality combined with the fact that $\varepsilon$ reverses the sign of the fundamental class. The second formula is a consequence of the first and the fact that $\varepsilon$ and $J$ anticommute. The third formula is a consequence of the first and the second.

We are now ready to prove Theorem 6.1 by a direct calculation.

LEMMA 6.2. If $k$ is even

(i) $p_{+} \circ \mathrm{Sh} \Theta_{\gamma}^{+}=i(-1)^{k-1} 2^{2 k-2}\left(\Omega_{\gamma}+\Omega_{\gamma^{\prime}}\right)$,

(ii) $p_{-} \circ \mathrm{Sh} \Theta_{\gamma}^{-}=(-1)^{k-1} 2^{2 k-2}\left(\Omega_{\gamma}-\Omega_{\gamma^{\prime}}\right)$.

If $k$ is odd

(iii) $p_{-} \circ \mathrm{Sh} \Theta_{\gamma}^{+}=i(-1)^{k-1} 2^{2 k-2}\left(\Omega_{\gamma}+\Omega_{\gamma^{\prime}}\right)$,

(iv) $p_{+} \circ \mathrm{Sh} \Theta_{\gamma}^{-}=(-1)^{k-1} 2^{2 k-2}\left(\Omega_{\gamma}-\Omega_{\gamma^{\prime}}\right)$.

PROOF. We prove (iii) and leave the rest to the reader.

$$
\begin{aligned}
p_{-} \circ \operatorname{Sh} \Theta_{\gamma}^{+} & =\frac{1}{2} p_{-}\left(\operatorname{Sh} \Theta_{\gamma}+\operatorname{Sh} \Theta_{\gamma^{\prime}}\right) \\
& =(-1)^{k-1} 2^{2 k-2}\left\{p_{-}\left(\Phi_{\gamma}+i \Omega_{\gamma}\right)+p_{-}\left(\Phi_{\gamma^{\prime}}+i \Omega_{\gamma^{\prime}}\right)\right\} \\
& =(-1)^{k-1} 2^{2 k-3}\left\{\left(\Phi_{\gamma}+i \Omega_{\gamma}-\varepsilon \Phi_{\gamma}-i \varepsilon \Omega_{\gamma}\right)\right. \\
& \left.\quad+\left(\Phi_{\gamma^{\prime}}+i \Omega_{\gamma^{\prime}}-\varepsilon \Phi_{\gamma^{\prime}}-i \varepsilon \Omega_{\gamma^{\prime}}\right)\right\} .
\end{aligned}
$$

Substituting from Lemma 6.1 we obtain the lemma and as a consequence Theorem 6.1 is proved.

7. The intersection formula. We conclude this paper by explaining how the period formula, Theorem 3 of S. Katok [8], follows from a computation of intersection products in Eichler-Shimura homology theory. This period formula expresses the quantity $I$ defined by

$$
I=\frac{1}{2 i}\left\{\left(\left(\Theta_{\gamma_{0}}, \Theta_{\gamma_{1}}\right)\right)_{2 k}-\left(\left(\Theta_{\gamma_{0}^{\prime}}, \Theta_{\gamma_{1}^{\prime}}\right)\right)_{2 k}\right\}=\operatorname{Im}\left(\left(\Theta_{\gamma_{0}}, \Theta_{\gamma_{1}}\right)\right)_{2 k}
$$

as the sum over the points $\left\{p_{i}\right\}$ of $\gamma_{0} \cap \gamma_{1}$ of terms involving elementary multiples of $\mu_{i} P_{k-1}\left(\cos \theta_{i}\right)$ where $P_{k-1}(t)$ is the $(k-1)$ th Legendre polynomial, $\theta_{i}$ is the angle of intersection at $p_{i}$ and $\mu_{i}$ is the intersection multiplicity (hence $\mu_{i}= \pm 1$ ) of the intersection $p_{i}$ of two oriented curves $\left[\gamma_{0}\right]$ and $\left[\gamma_{1}\right]$.

We begin by simplifying $I$.

LEMMA 7.1 .

$$
I=(-1)^{k-1} 2^{2 k-2} \int_{M} \Omega_{\gamma_{0}} \wedge \Omega_{\gamma_{1}}
$$


PROOF.

$$
\begin{aligned}
I & =\operatorname{Im}\left(\left(\Theta_{\gamma_{0}}, \Theta_{\gamma_{1}}\right)\right)_{2 k}=2^{-(2 k-1)}\left(\left(\operatorname{Sh} \Theta_{\gamma_{0}}, \operatorname{Sh} \Theta_{\gamma_{1}}\right)\right) \\
& =2^{2 k-1} \operatorname{Im}\left(\left(\Xi_{\gamma_{0}}, \Xi_{\gamma_{1}}\right)\right) .
\end{aligned}
$$

An easy computation gives

$$
\operatorname{Im}\left(\left(\Xi_{\gamma_{0}}, \Xi_{\gamma_{1}}\right)\right)=(-1)^{k-1} \frac{1}{2} \int_{M} \Omega_{\gamma_{0}} \wedge \Omega_{\gamma_{1}} .
$$

With this the lemma is proved.

Since Poincare duality makes the exterior product of differential forms correspond to the intersection product of the corresponding cycles we obtain

$$
I=(-1)^{k-1} 2^{2 k-2}\left[\gamma_{0}\right] \otimes \sigma_{\gamma_{0}} \cdot\left[\gamma_{1}\right] \otimes \sigma_{\gamma_{1}},
$$

where the product on the right-hand side is the intersection product of cycles with coefficients. Recall that $\sigma_{\gamma_{0}}$ and $\sigma_{\gamma_{1}}$ are parallel sections of $\tilde{E}^{*}$ corresponding to the $\xi_{\gamma_{0}}$ and $\xi_{\gamma_{1}}$ restricted to $\left[\gamma_{0}\right]$ and $\left[\gamma_{1}\right]$ respectively (see $\S 2$ ).

By the definition we have

$$
\left[\gamma_{0}\right] \otimes \sigma_{\gamma_{0}} \cdot\left[\gamma_{1}\right] \otimes \sigma_{\gamma_{1}}=\sum_{p_{i} \in\left[\gamma_{0}\right] \cap\left[\gamma_{1}\right]} \mu_{i}\left(\sigma_{\gamma_{0}}\left(p_{i}\right), \sigma_{\gamma_{1}}\left(p_{1}\right)\right) .
$$

Here $\mu_{i}$ is the intersection multiplicity, $\mu_{i}=\operatorname{sgn}\left(\sin \theta_{i}\right)$, and $\left(\sigma_{\gamma_{0}}\left(p_{i}\right), \sigma_{\gamma_{1}}\left(p_{i}\right)\right)$ is the coefficient contribution which we are going to compute.

Let $\left[\hat{\gamma}_{0}\right]$ and $\left[\hat{\gamma}_{1}\right]$ denote the lifts of $\left[\gamma_{0}\right]$ and $\left[\gamma_{1}\right]$ into $\mathbf{H}$ which intersect in a point denoted $\hat{p}_{i}$. If we consider oriented geodesics $\left[\hat{\gamma}_{0}\right]$ and $\left[\hat{\gamma}_{1}\right]$ in $\mathbf{H}$ as infinite cycles with coefficients $\left[\hat{\gamma}_{0}\right] \otimes \xi_{\hat{\gamma}_{0}}$ and $\left[\hat{\gamma}_{1}\right] \otimes_{\hat{\gamma}_{1}}$ with $\xi_{\hat{\gamma}_{0}}, \xi_{\hat{\gamma}_{1}} \in E^{*}$, then $\sigma_{\gamma_{0}}\left(p_{i}\right)=\xi_{\hat{\gamma}_{0}}$ and $\sigma_{\gamma_{1}}\left(p_{i}\right)=\xi_{\hat{\gamma}_{1}}$, and in order to compute the intersection product we need a formula for $\left(\xi_{\hat{\gamma}_{0}}, \xi_{\hat{\gamma}_{1}}\right)$. Since the form (, $)$ is $P S L_{2}(\mathbf{R})$-invariant we can always bring the geodesic $\left[\hat{\gamma}_{0}\right]$ into the $y$-axis by conjugation. Then

$$
\xi_{\hat{\gamma}_{0}}=D_{0}^{k-1 / 2}\left(-\operatorname{sgn} \operatorname{tr} \gamma_{0}\right)^{k-1} u^{k-1} v^{k-1},
$$

where $D_{0}=\left(\operatorname{tr} \gamma_{0}\right)^{2}-4\left(\right.$ see $\left[8\right.$, p. 471]). Let $\xi_{\hat{\gamma}_{1}}=\left(A u^{2}+B u v+C v^{2}\right)^{k-1}$. We have $D_{1}=B^{2}-4 A C=\left(\operatorname{tr} \gamma_{1}\right)^{2}-4>0$, and $\left[\hat{\gamma}_{1}\right]$ intersects $\left[\hat{\gamma}_{0}\right]$ if and only if $A C>0$. The form (, ) has a very simple matrix $W$ in the basis $\left\{u^{i} v^{2 k-2-i}\right\}$

$$
W=\left(w_{i j}\right), \quad \text { where } \begin{cases}w_{i j}=0 & \text { if } i+j \neq 2 k \\
w_{i j}=(-1)^{i-1}\left(\begin{array}{c}
2 k-2 \\
i-1
\end{array}\right)^{-1} & \text { if } i+j=2 k\end{cases}
$$

Therefore

$$
\begin{aligned}
\left(\xi_{\hat{\gamma}_{0}}, \xi_{\hat{\gamma}_{1}}\right)= & D_{0}^{k-1 / 2}\left(-\operatorname{sgn} \operatorname{tr} \gamma_{0}\right)^{k-1}\left(\begin{array}{c}
2 k-2 \\
i-1
\end{array}\right)^{-1}(-1)^{k-1} \\
& \cdot\left\{\text { coefficient of } u^{k-1} v^{k-1} \text { in } \xi_{\hat{\gamma}_{1}}\right\} .
\end{aligned}
$$

The computation of the above coefficient is done in the following combinatorial lemma. 
LEMMA 7.2. The coefficient of $u^{n} v^{n}$ in $\left(A u^{2}+B u v+C v^{2}\right)^{n}$ is equal to $D_{1}^{n / 2} P_{n}\left(B / \sqrt{D_{1}}\right)$, where $D_{1}=B^{2}-4 A C$ and $P_{n}$ is the $n$th Legendre polynomial.

PROOF. The standard formula

$$
P_{\nu}(z)=z^{\nu} F\left(-\frac{\nu}{2}, \frac{1-\nu}{2} ; 1 ; 1-\frac{1}{z^{2}}\right)
$$

(cf. Bateman $[\mathbf{1}, 3.2(24)]$ ) expressing the Legendre function of the first kind in terms of the hypergeometric function becomes a polynomial identity

$$
\begin{aligned}
P_{n}(z) & =z^{n} F\left(-\frac{n}{2}, \frac{1-n}{2} ; 1 ; 1-\frac{1}{z^{2}}\right) \\
& =z^{n} \sum_{0 \leq j \leq n / 2} \frac{n !}{j !^{2}(n-2 j) !}\left(\frac{z^{2}-1}{4 z^{2}}\right)^{j}
\end{aligned}
$$

where $\nu=n$ is a nonnegative integer. Substituting $z=B / \sqrt{D_{1}}$ gives

$$
\begin{aligned}
D_{1}^{n / 2} P_{n}\left(\frac{B}{\sqrt{D_{1}}}\right) & =\sum_{0 \leq j \leq n / / 2} \frac{n !}{j !^{2}(n-2 j) !} B^{n-2 j}(A C)^{j} \\
& =\left\{\text { coefficient of } u^{n} v^{n} \text { in }\left(A u^{2}+B u v+C v^{2}\right)^{n}\right\} .
\end{aligned}
$$

With this the lemma is proved.

We have $B / \sqrt{D_{1}}=\left(-\operatorname{sgn} \operatorname{tr} \gamma_{1}\right) \cos \theta_{i}$ (see $[8$, p. 478]) and therefore

$$
\begin{aligned}
I= & D_{0}^{k-1 / 2} D_{1}^{k-1 / 2}\left(\operatorname{sgn} \operatorname{tr} \gamma_{0} \operatorname{tr} \gamma_{1}\right)^{k-1} \\
& \cdot\left(\begin{array}{c}
2 k-2 \\
k-1
\end{array}\right)^{-1} 2^{2 k-2} \sum_{p_{i} \in\left[\gamma_{0}\right] \cap\left[\gamma_{1}\right]} \mu_{i} P_{k-1}\left(\cos \theta_{i}\right) .
\end{aligned}
$$

\section{REFERENCES}

1. H. Bateman, Higher transcendental functions, Vol. I, McGraw-Hill, New York, 1953-55.

2. A. Borel and N. Wallach, Continuous cohomology, discrete subgroups and representations of reductive groups, Ann. of Math. Studies, no. 94, Princeton Univ. Press, Princeton, N. J., 1980 .

3. K. Brown, Cohomology of groups, Graduate Texts in Math., vol. 87, Springer-Verlag, Berlin and New York, 1972.

4. W. Goldman, The symplectic nature of fundamental groups of surfaces, Adv. in Math. 54 (1984), 200-225.

5. W. Goldman and J. Millson, Eichler-Shimura homology and the finite generation of cusp forms by hyperbolic Poincaré series, Duke Math. J. (to appear).

6. P. Griffiths and J. Harris, Principles of algebraic geometry, Wiley, New York, 1978.

7. D. Johnson and J. Millson, Deformation spaces associated to compact hyperbolic mani folds, Discrete groups in geometry and analysis, Birkhäuser (to appear).

8. S. Katok, Closed geodesics, periods and arithmetic of modular forms, Invent. Math. 80 (1985), 469-480.

9. S. Kudla and J. Millson, Harmonic differentials and closed geodesics on a Riemann surface, Invent. Math. 54 (1979), 193-211.

10. Y. Matsushima and S. Murakami, On vector bundle valued harmonic forms and automorphic forms on symmetric spaces, Ann. of Math. (2) 78 (1963), 365-416. 
11. S. Murakami, Cohomology groups of vector-valued forms on symmetric spaces, Lecture Notes, University of Chicago, 1966.

12. M. Raghunathan, Discrete subgroups of Lie groups, Ergebnisse Math. Grenzgeb., vol. 68, Springer-Verlag, Berlin and New York, 1972.

13. G. Shimura, Sur les intégrales attachées aux formes automorphes, J. Math. Soc. Japan 11 (1959), 291-311.

Department of Mathematics, University of California at Los Angeles, Los ANGELES, CALIFORNiA 90024

Current address (Svetlana Katok): Department of Mathematics, University of California at San Diego, La Jolla, California 92093 